\title{
The First Year of Life
}

\section{INFANTS OF LOW BIRTH WEIGHT}

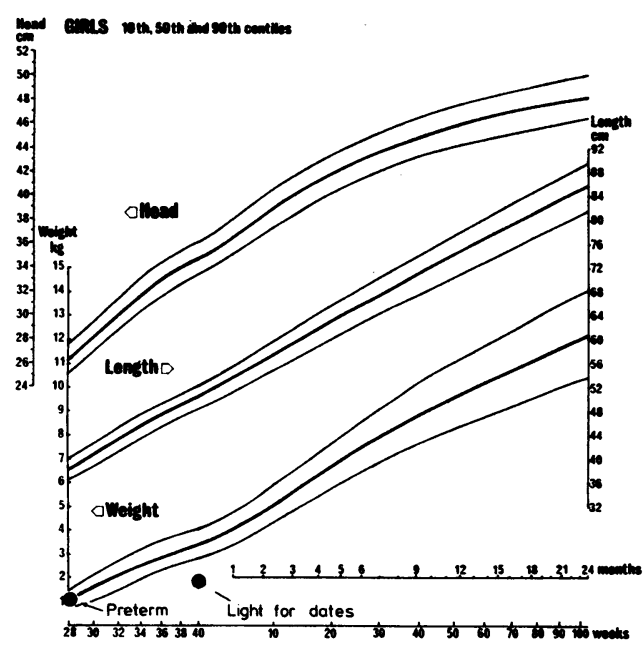

Low birth weight infants weigh $2500 \mathrm{~g}$ or less at birth. Infants may be small at birth owing to a short gestation period (born too early) or because of a retarded growth rate. When the period of gestation is less than 37 completed weeks the infant is called preterm. A baby with retarded growth rate is "light-for-dates" and may be either malnourished or rarely hypoplastic (for example, an infant with a chromosome abnormality). Some babies who are preterm are also light-for-dates.

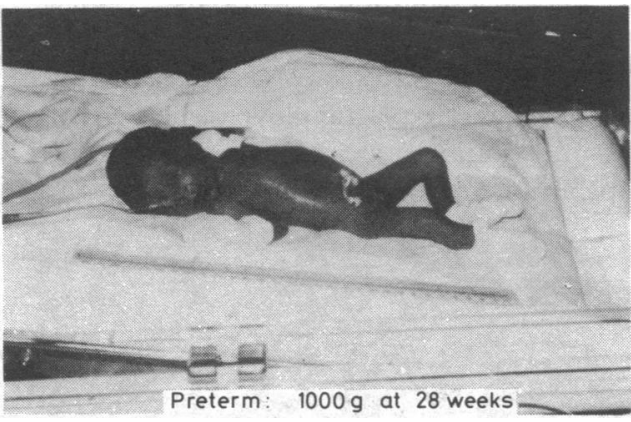

The length of gestation, as calculated from the date of the mother's last menstrual period, can be confirmed by a detailed neurological examination of the infant, as the development of the central nervous system is related to the gestational age. Scoring systems using both the neurological development and specific external features can be used to estimate the gestational age within an accuracy of about a week. Detecting retardation in growth of the fetus is difficult, but palpation of the fetus can now be supplemented by serial measurements of the fetal skull, abdominal girth, and liver size by ultrasound. Most units consider that infants with a birth weight below the 10th centile for the gestational age are light-for-dates, although a more accurate definition is below the 5th centile with an adjustment made for maternal size.

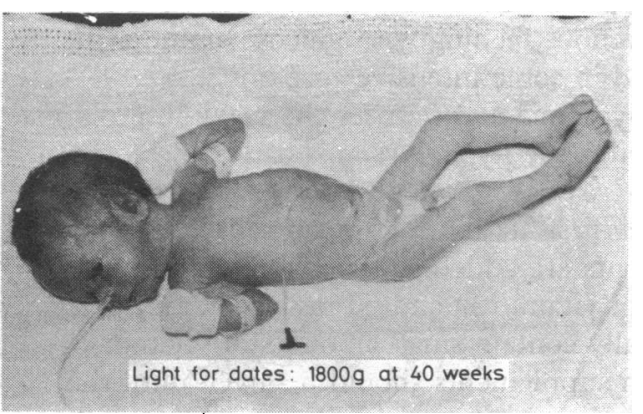

Recent ultrasound studies have shown that when intrauterine malnutrition starts early in pregnancy the head circumference and weight are in proportion with each other, but when malnutrition starts late in pregnancy the head is disproportionately large owing to relatively normal growth of the brain.

The preterm infant is especially prone to developing hypothermia, the respiratory distress syndrome, infection, and intraventricular or subarachnoid haemorrhage. The light-for-dates infant is particularly prone to hypothermia, hypoglycaemia, and hypocalcaemia. 


\section{Temperature}

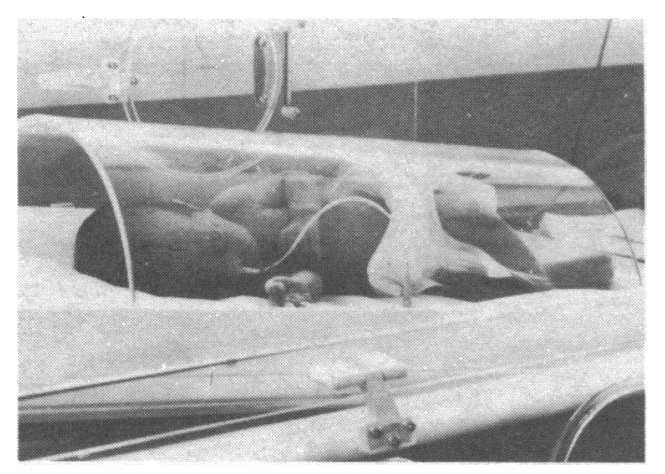

\section{Infection}

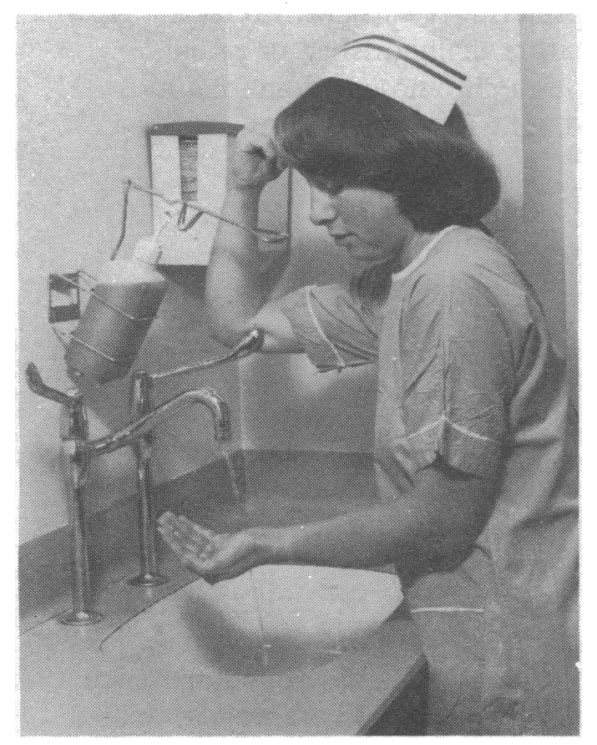

\section{Feeding}

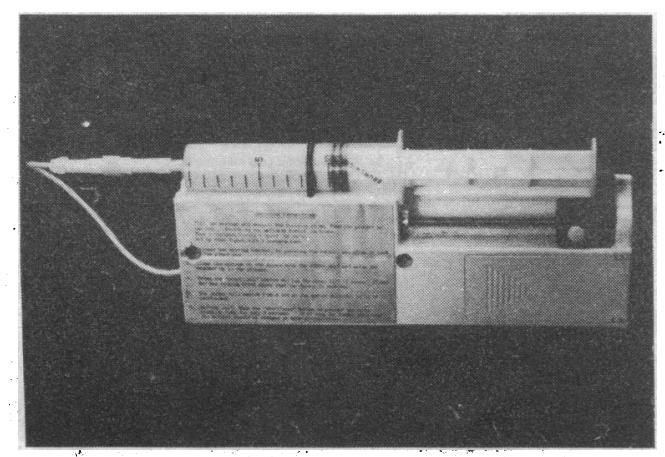

Small infants become hypothermic quickly. Heat loss may be considerable because they have a large surface area in relation to body weight and they are also deficient in subcutaneous fat, which provides insulation. They also lack "brown fat," which is usually present in a full-term baby and can be metabolised rapidly to produce heat.

Hypothermia is associated with a raised metabolic rate and increased energy consumption; to prevent it small infants should be kept in a high constant environmental temperature. Excessive heat loss by radiation can be minimised by an additional tunnel of perspex, a heat shield, placed immediately over an infant in an incubator. The dangers of hypothermia can be reduced by carrying out resuscitation under a heat lamp or radiant heat canopy, bearing in mind the danger of burns if the lamp is too close. When an infant is transferred from one hospital to another for special care there is a danger of hypothermia. A swaddler made of aluminium foil has been designed to prevent this and is commercially available, but wrapping the baby with Gamgee wool is also satisfactory.

The portable incubator must be kept warm continuously and ready for immediate use.

Nurses who are scrupulous about washing their hands are probably the most important factor in preventing cross-infection. In many units medical and nursing staff no longer wear masks and gowns, though a separate plastic apron or waterproof gown should be used for nursing each infant, mainly to prevent soiling the nurses' clothes.

Scrupulous washing of the hands should be carried out before and after touching each infant. Washing with soap and water is adequate, although many units use a disinfectant soap solution. Scrubbing with a brush is unnecessary. Although this principle is simple it may be difficult to ensure that it is carried out. Proper use of elbow taps is often difficult because of their poor design. Using disposable plastic gloves when changing infants' napkins may reduce cross-infection.

Ideally, any infant who develops an infection should be barrier nursed in a separate cubicle.

Preterm infants have poor sucking and cough reflexes, and methods of feeding must prevent aspiration into the lungs. Frequent tube feeds of small volumes or continuous intragastric feeds avoid sudden falls in arterial oxygen concentrations and apnoeic attacks which are associated with abdominal distention. In many units transpyloric feeding using a weighted tube which passes into the jejunum has been used successfully, but it is technically more difficult. Intravenous feeding with glucose amino-acid solutions and lipids is being used in some intensive care units.

Early feeding prevents hypoglycaemia and reduces the maximum plasma bilirubin concentrations. Asymptomatic hypoglycaemia can be detected early by performing regular Dextrostix tests on all babies with low birth weights every three hours for the first $\mathbf{4 8}$ hours of life. From the 2 nd week onwards vitamin supplements are added so that the infant receives an additional dose of 400 units of vitamin $D$ and $50 \mathrm{mg}$ of vitamin C daily. Vitamin preparations usually contain small amounts of vitamin B complex and vitamin A. Vitamin supplements should be given until the age of 2 years and additional iron supplements until the age of 6 months.

Dr H B Valman, MD, FRCP, is consultant paediatrician, Northwick Park Hospital and Clinical Research Centre, Harrow. 